\title{
COLOURED FRUITS AND VEGETABLES FOR HEALTHY BODY
}

\author{
Amrita Khatri
}

M.J.B. Govt. P. G. College, Moti Tabela, Indore

Email: amritakhatri53@gmail.com

Fruits and vegetables are extremely essential to our healthiness because they are whole foods, produced by natural world. They are rich in a large amount of nutrients, as they possess fiber, vitamins, and enzymes .Each different color fruit and vegetable contains unique health components that are essential to our health.

Green color is very crucial in diet. Green vegetables contain chlorophyll, calcium, folate, vitamin $\mathrm{C}$, calcium, and beta-carotene. The green fruit and vegetables are also rich in phytochemicals which are important in keeping the healthy body e.g. spinach has carotinoids, lutein and zeaxanthin .The antioxidant properties possessed by broccoli are good to defend the eyes by keeping the retina healthy and powerful. Peas and lima beans are also good sources .The green Fruits and vegetables are also encumbered with necessary minerals and fibers. The green vegetables are loaded with cancer blocking chemicals like sulforaphanes, isosynate and indols which reduces the stroke of carcinogens. They are also associated with reduction of cataract and age related muscular degeneration, thus also controls blindness. The nutrients found in these vegetables reduce cancer risks, lower blood pressure, LDL cholesterol levels, normalize digestion time, support retinal health and vision, fight harmful free-radicals, and boost immune system activity. Green colour of fruits and vegetables enhances calcium absorption, boosts mental alertness and helps in cell regulation.

COMPOUNDS RESPONSIBLE FOR GREEN COLOUR - The green fruits and vegetables have compounds that give them colour, are called glucosinolates. This is a large group of sulphurcontaining amino acid derivatives. Certain glucosinolates and their breakdown products may reduce the prevalence of certain types of cancer. The anticarcinogenic effect of glucosinolates is explained by the activation of enzymes involved in the detoxification of carcinogens, inhibition of enzymes modifying steroid hormone metabolism and protection against oxidative damage (Hounsome, et al.).

Glucosinolates are found in broccoli, cauliflower, Brussels sprouts and cabbage (Heber, et al.).High amount of iron and folic acid is present in green leafy vegetables. The ascorbic acid or vitamin $\mathrm{C}$ present in them facilitates iron absorption (Cámara, et al.). The chlorophyll is green in color .This pigment facilitates the plant to continue photosynthesis, thus convert solar energy and carbon dioxide into chemical energy in the form of carbohydrates and oxygen. This is a process essential for life. The configuration of chlorophyll is very complex, so one can simply say that it contains a big ring with a magnesium atom in the center. Amazingly, the structure of hemoglobin (the carrier of oxygen in our blood) is very similar to chlorophyll, though it has an atom of iron instead of magnesium in its center. 
RED FRUITS AND VEGETABLES - In red/ pink fruits and vegetables, a powerful antioxidant is present called lycoprotein .These are supposed and studied for their ability to defend the body against heart and lung diseases and also some cancers e.g. Prostate Cancer. The caroteinoids are also supposed to be effective scavenger of gene -damaging free radicals. Red fruits and vegetables get their color from lycopene and anthocyanins. However, anthocyanin is a powerful antioxidant that assures for reducing the risk of pregnancy-related high blood pressure and for treating gingivitis. Strawberries, Red rasberries and Tomatoes are the best sources of lycopene. In addition to lycopene, ellagic acid, quercetin, and hesperidin are few nutrients present in them, which reduce the risk of prostate cancer, lower blood pressure, reduce tumor growth and LDL cholesterol levels, scavenge harmful free-radicals, and support join tissue in arthritis cases. Red cabbage is sulfur-rich and colorful (with 36 different anthocyanins). Red colour also maintains consistent energy levels.

COMPOUNDS RESPONSIBLE FOR RED COLOUR - The red colour of fruits and vegetables such as tomato, watermelon, or grapefruit is due to the compound lycopene (also belonging to the carotenoids, like $\alpha$ - and $\beta$-carotene), and the red-purple colour of grapes, berries, raspberries and cranberries is caused by anthocyanins (Heber, D. et al.). Anthocyanins have antioxidant properties that have been demonstrated in both in vitro and in vivo. They belong to the biggest group of phenolic compounds, called flavonoids. The number of hydroxyl groups, the nature and number of sugars, and the position of these groups separate anthocyanins from the rest of the flavonoids. It has been discovered that anthocyanins are absorbed from the diet without being modified. Experiments suggested that anthocyanins are very important in preventing carcinogenesis and mutagenesis (Lazzè, et al.).

Lycopene is one of the first carotenoids that occur during the synthesis of this family of compounds, thus constituting the base to synthesize the others. Unlike $\alpha$ - and $\beta$-carotene, lycopene is not a vitamin A precursor. It is a carotenoid with a simple aliphatic chain structure of 40 carbon atoms and many conjugated double bonds (de Carlos, P.). It has a very strong antioxidant capacity, plays a role in cell communication, and there is experimental evidence that it protects against prostate cancer, cardiovascular disease, and damage from ultraviolet light exposure and tobacco smoke (Aguilera Garca, C. M., et al.). The red colour of tomato is because of the carotenoid lycopene, which contains 11 conjugated carbon-carbon double bonds which can be counted. This compound is generated by the plant to protect itself from the air oxidation. So it is a high-quality antioxidant useful for us too, protecting our cells against the action of free radicals (potent oxidants), which are mainly responsible for cardiovascular diseases, cancer and aging. However, some red fruits and vegetables have relatively low vitamin $\mathrm{C}$.

ORANGE FRUITS AND VEGETABLES - It is also one of the important colour which should be added to our diet .The common orange coloured fruits and vegetables are mangoes, carrots, apricots et..They contain alpha, beta-carotene, which are supposed to be responsible for monitoring immune system, being an effective antioxidant. It also protects the skin against free radical damage 
and promotes repair of damaged DNA. It also contains Vitamin C. The orange fruits and vegetables also posses folate which are often present in leafy green vegetables also. The Vitamin B is also present in some of orange group fruits and vegetables which help to control birth defects and heart diseases. The orange and yellow colour are the result of carotenoids. Three of the carotenoids -alpha carotene, beta carotene and beta cryptoxanthin -- provide a different advantage than other phytonutrients: Our body converts them into vitamin A. Two other carotenoids, lutein and zeaxanthin, work only as antioxidants in eyes, where they absorb blue light before it damages the retina. Some of the top sources of carotenoids include carrots, sweet potatoes and pumpkin.. They contain beta-carotene, zeaxanthin, flavonoids, lycopene, potassium, and vitamin $\mathrm{C}$. These nutrients reduce age-related macula degeneration and the risk of prostate cancer, lower LDL cholesterol and blood pressure, promote collagen formation and healthy joints, fight harmful free radicals, encourage alkaline balance, and work with magnesium and calcium to build healthy bones. Orange/yellow coloured fruits includes oranges, peaches, papaya and nectarines, which provide beta cryptothanxin. They support intracellular communication and may help prevent heart disease.

COMPOUNDS RESPONSIBLE FOR ORANGE COLOUR - The orange colour in fruits and vegetables such as carrot, mango, or pumpkin, are due to the carotenoids, $\alpha$ - $\mathrm{y} \beta$-carotene. Carotenoids belong to the class of isoprenoid lipids and derive their colour from conjugated carbon-carbon double bonds in the chemical structure. Carotenoids give fruits and vegetables their bright orange, red, or yellow colors, and are a source of dietary vitamin A. between 40 and 50 carotenoids are present in our diets that can be absorbed, metabolized, (Aguilera, et al.). In particular, $\alpha$ and $\beta$-carotene are very important in the diet because they are vitamin A precursors, that's why they are also called provitamin A. Vitamin A is involved in hormone synthesis, regulation of cell growth and differentiation, and immune responses (Hounsome, N. et al.). Prior studies report that oxidative stress plays a role in the development of ALS. Further studies have shown that individuals with high intake of antioxidants, such as vitamin E, have a reduced ALS risk. Because vitamin $\mathrm{C}$ or carotenoids are also antioxidants, researchers examined their relation to ALS risk. They maintain bone density, support energy production and helps in protecting cells.

YELLOW FRUITS AND VEGETABLES - Yellow fruits and vegetables have same advantages as orange group i.e. pineapple has Vitamin $\mathrm{C}$, manganese and natural enzyme brome lain corn and pears having high contents of fiber. The common yellow items include oranges, peaches, papaya etc. They contain beta cryptothanxin and may help in preventing diseases. Fruits and vegetables of these colors are rich in potassium, vitamin c, lycopene and beta-carotene. They help in promoting the formation of collagen formation, formation of bones, lowering cholesterol and promote the balance of alkaline in our body

COMPOUNDS RESPONSIBLE FOR YELLOW COLOUR - The flavonoids, as mentioned above the largest group of phenolic compounds, together with $\beta$-cryptoxanthin are responsible for the light orange to yellow colour of fruits such as peach, papaya or orange (Heber, D. et al.). 
Dietary flavonoids possess antiviral, anti-inflammatory, antihistamine and antioxidant properties. They have been reported to inhibit lipid peroxidation, scavenge free radicals, chelate iron and copper ions, and to modulate cell signaling pathways. Production of peroxides and free radicals has been linked to cancer, aging, ischemic injury, and neurodegenerative diseases such as Parkinson's and Alzheimer's disease. Flavonoids protect low-density lipoprotein cholesterol from being oxidized, thus preventing the formation of atherosclerotic plaques in the arterial wall. B-cryptoxanthin like $\beta$-carotene or lycopene is a carotenoid, and as such has an important function as biologic antioxidant, protecting cells and tissues from oxidative damage (Hounsome. et al.). In carrots, the beta carotene pigment, with 9 linear conjugated double bonds is present which is lesser than in lycopene so they are not red but orange (smaller wavelength than red, check it in the spectrum picture). This complex is also a potent antioxidant and besides it's transformed in our body into vitamin A, very important for the maintenance of healthy skin, good vision and a robust immune system.

BLUE AND PURPLE FRUITS AND VEGETABLES - Blue and purple Plant pigments called anthocyanins impart shades of, as well as red. The anthocyanins belong to a larger family of phytochemicals called flavonoids. Like all flavonoids, anthocyanins are antioxidants. They may also help prevent cancer by inhibiting the growth of cancerous cells or by stimulating the release of cancer-killing substances. Researchers from the Harvard School of Public Health reported that consuming two to five servings a week of anthocyanin-rich fruits may lower the risk of Type 2 diabetes, according to the April 2012 issue of "The American Journal of Clinical Nutrition." Blueberries, blackberries, grapes, and juices or wines made from blue and purple fruits contain anthocyanins.

WHITE FRUITS AND VEGETABLES - A group of pigments called anthoxanthins is responsible for shades of white to yellow. These phytochemicals belong to the family of antioxidant flavonoids. Some anthoxanthin-containing foods have other potent phytochemicals that aren't pigments but provide benefits, such as allicin in garlic and onions that helps lower cholesterol. The most abundant plant color comes from green chlorophyll. While it has antioxidant properties and may help prevent colon cancer.

COMPOUNDS RESPONSIBLE FOR WHITE COLOUR - Contain nutrients such as betaglucans, EGCG, SDG, and lignans that provide powerful immune boosting activity. These nutrients also activate natural killer $\mathrm{B}$ and $\mathrm{T}$ cells, reduce the risk of colon, breast, and prostate cancers, and balance hormone levels, reducing the risk of hormone-related cancers. The onion family contains allicin, which has antitumor properties. Other foods in this group contain antioxidant flavonoids like quercetin and kaempferol. Dr. Heber includes white wine in this category. 


\section{INTERNATIONAL JOURNAL of RESEARCH -GRANTHAALAYAH \\ A knowledge Repository}

Art

BLACK FRUITS AND VEGETABLES - According to Purdue University, dark pigmented vegetables have reliable measure of antioxidant value. Black, or wild, rice; black lentils; and black beans all provide high levels of antioxidants relative to their lighter-colored counterparts. Black rice contains 70 percent more protein and twice as much copper as brown rice, though it is lower in manganese and zinc than its fairer-hued relative,. However, white beans provide more iron, potassium and protein than black.

\begin{tabular}{|l|l|l|}
\hline Colour & Phytochemical & Fruits and vegetables \\
\hline Green & Glucosinolates & Broccolo, kale \\
\hline Orange & $\alpha$ - and $\beta$-carotene & Carrot, mango, pumpkin \\
\hline Red & Lycopene & Tomato \\
\hline Red-purple & Anthocyanins & Grapes, blackberries, raspberries, blueberries \\
\hline Orange-yellow & Flavonoids & Honeydew melon, peach, papaya, orange, tangerine \\
\hline Yellow-green & Lutein and zeaxanthin & Spinach, corn, avocado, melon \\
\hline
\end{tabular}

Source: Heber, D. et al.

\section{CONCLUSION}

Eating plenty of coloured vegetables and fruits helps to prevent heart disease and strokes, diverticulitis, control blood pressure, prevent some types of cancers, and guards against cataract and macular degeneration or vision loss.

\section{REFERANCES}

1 Cámara Hurtado, M., de Cortes Sánchez Mata, M., Torija Isasa, M. (2003). Frutas y verduras, fuente de salud. Instituto de Salud Pública. Consejería de Sanidad y Consumo.

2 Heber, D., Bowerman, S. (2001). Applying Science to Changing Dietary Patterns. AmericanInstitute for Cancer Research 11th Annual Research Conference on Diet, Nutrition and Cancer

3 Lazzè, M. C., Savio, M., Pizzala, R., Cazzalini, O., Perucca, P., Scovassi, A.I., Stivala, L. A. y Bianchi, L. (2004). Anthocyanins induce cell cycle perturbations and apoptosis in different human cell lines. Carcinogenesis vol. $25 n^{\circ} 8$ p.1427-1433.

4 Hounsome, N., Hounsome, B., Tomos, D., y Edwards-Jones, G. (2008.) Plant Metabolites and Nutritional Quality of Vegetables. Journal Food of Science. Vol.73, Nr. 4, p. 48-62. 